\title{
WEIGHTED NORM INEQUALITIES FOR MAXIMAL FUNCTIONS FROM THE MUCKENHOUPT CONDITIONS
}

\author{
Y. RAKOTONDRATSIMBA
}

\begin{abstract}
For some pairs of weight functions $u, v$, which satisfy the wellknown Muckenhoupt conditions, we derive the boundedness of the maximal fractional operator $M_{s}(0 \leq s<n)$ from $L_{v}^{p}$ to $L_{u}^{q}$ with $q<p$.
\end{abstract}

\section{Introduction}

Let $u, v$ weight functions on $\mathbb{R}^{n}, n \geq 1$ (i.e. nonnegative locally integrable functions). The fractional maximal operator $M_{s}(0 \leq s<n)$ is given by

$$
\left(M_{s} f\right)(x)=\sup \left\{|Q|^{\frac{s}{n}-1} \int_{Q}|f| ; \quad Q \text { a cube with } Q \ni x\right\}
$$

Throughout this paper $Q$ will denote a cube with sides parallel to the co-ordinate planes.

Let $1<p, q<\infty$, with $\frac{1}{p}-\frac{1}{q} \leq \frac{s}{n}$. It is fundamental in analysis to give a characterization of the pairs of weights $(u, v)$ which satisfy

(0) $\left\|M_{s} f\right\|_{L_{u}^{q}} \leq C\|f\|_{L_{v}^{p}}$ for all functions $f, C=C(s, n, p, q, u, v)>0$.

Here $\|g\|_{L_{w}^{r}}$ denotes $\left(\int_{\mathbb{R}^{n}}|g|^{r} w d x\right)^{\frac{1}{r}}$, with $d x$ the Lebesgue measure on $\mathbb{R}^{n}$.

In the case of $1<p \leq q<\infty$ Sawyer [Sa2] showed that the inequality (0) holds if and only if $(u, v) \in S(s, n, p, q)$ i.e

$$
\left\|\left(M_{s} v^{-\frac{1}{p-1}} \mathbb{I}_{Q}\right) \mathbb{I}_{Q}\right\|_{L_{u}^{q}} \leq C\left\|v^{-\frac{1}{p-1}} \mathbb{I}_{Q}\right\|_{L_{v}^{p}}=\left\|\mathbb{I}_{Q}\right\|_{L^{p}{ }^{-\frac{1}{p-1}}}<\infty
$$


for all cubes $Q$, here $S=S(s, n, p, q, u, v)>0$. A known necessary but not sufficient condition for $(0)[\mathrm{Mu}]$ is $(u, v) \in A(s, n, p, q)$ i.e

$$
|Q|^{\frac{s}{n}+\frac{1}{Q}-\frac{1}{p}}\left(\frac{1}{|Q|} \int_{Q} u\right)^{\frac{1}{Q}}\left(\frac{1}{|Q|} \int_{Q} v^{-\frac{1}{p-1}}\right)^{1-\frac{1}{p}} \leq A \text { for all cubes } Q
$$

with $A=A(s, n, p, q, u, v)>0$.

As we will recall in Section 2, this condition is verified more easily than the first one. Pérez $[\mathbf{P e}]$ (see also [Sa1]) proved that $(u, v) \in A(s, n, p, q)$ implies the inequality ( 0 ) whenever $d \sigma=v^{-\frac{1}{p-1}} d x \in A_{\infty}$ i.e. for some $\delta>0$ :

$$
\frac{|E|_{\sigma}}{|Q|_{\sigma}} \leq\left(\frac{|E|}{|Q|}\right)^{\delta} \text { for all cubes } Q \text { and for all measurable sets } E \subset Q
$$

here $|E|_{\sigma}$ denotes $\int_{Q} \sigma$. In fact the equivalence between $(0)$ and $(u, v) \in$ $A(s, n, p, q)$ is also valid with a weaker condition on $d \sigma$, for instance in [Ra3] it was proved that it is sufficient $d \sigma \in B_{\delta}$ i.e.

$$
\frac{\left|Q^{\prime}\right|_{\sigma}}{|Q|_{\sigma}} \leq\left(\frac{\left|Q^{\prime}\right|}{|Q|}\right)^{\delta} \text { for all cubes } Q, Q^{\prime} \text { with } Q^{\prime} \subset Q
$$

with $\left[1-\frac{s}{n}\right] \leq \delta$. As we will see in Section 2 , measures $d \mu$ can be found such as $d \mu \in B_{\delta}$ but $d \mu \notin A_{\infty}$. The condition $\frac{1}{p}-\frac{1}{q} \leq \frac{s}{n}$ can be derived from the inequality (0) by the Lebesgue differentiation theorem. Hence for $s=0\left(M_{0}\right.$ is the Hardy-Littlewood maximal operator $)$, the inequality (0) must only considered for $q \leq p$. The case $p=q$ was studied by Muckenhoupt $[\mathbf{M u}]$ for $u=v$ and by Sawyer [Sa2] for general weights $u, v$. For $q<p$, a characterization of the pairwise of weights $(u, v)$ satisfying the inequality (0) was given by the author [Ra1]; but the condition used is difficult to check.

Therefore $1<q<p<\infty$ a natural question is: "does $(u, v) \in$ $A(s, n, p, q)$ imply (0) whenever $d \sigma \in A_{\infty}$. In this paper we give a positive answer with the additional assumptions $u d x \in B_{\nu}, v^{-\frac{1}{p-1}} d x \in B_{\rho}$ with $0<\nu, \rho$ and $\left[1-\frac{s}{n}\right]<\rho\left(1-\frac{1}{p}\right)+\nu \frac{1}{p}$.

We state our main result in Section 1. In Section 2 we give some useful remarks and observations about the weight condition $B_{\rho}$. The proof of our main result is in Section 3. A paper of Verbitsky [Ve] concerning the characterization of the problem (0) with $q<p$ and for general weights $u, v$ appeared when this manuscript was written.

Acknowledgement. The author would like to thank the referee for his helpful comments and suggestions. 


\section{The main result}

To include many classical maximal functions, we deal with the operator

$$
\left(M_{\Phi} f\right)(x)=\sup \left\{\Phi(Q)|Q|^{-1} \int_{Q}|f| ; \quad Q \text { a cube with } Q \ni x\right\}
$$

where $\Phi$ is a map defined on the set of cubes, taking its value in $] 0, \infty[$ and satisfying the following growth conditions:

$\mathcal{H}_{1}$ there is $C>0$ such as

$$
\Phi\left(Q_{1}\right) \leq C \Phi\left(Q_{2}\right) \text { for all cubes } Q_{1}, Q_{2} \text { with } Q_{1} \subset Q_{2} ;
$$

$\mathcal{H}_{2}$ there are $C_{1}, C_{2}>0, \lambda, \eta \geq 0$ such as

$$
C_{1} t^{n \lambda} \Phi(Q) \leq \Phi(t Q) \leq C_{2} t^{n \eta} \Phi(Q) \text { for all cubes } Q \text { and all } t \geq 1 \text {. }
$$

When $\Phi(Q)=1$ the Hardy-Littlewood maximal operator is obtained. The fractional maximal operator $M_{s}(0<s<n)$ is given by $\Phi(Q)=$ $|Q|^{\frac{s}{n}}$. Maximal operators connected to the Bessel potential operator $\left[\right.$ Ke-Sa] are defined by $\Phi(Q)=\int_{0}^{|Q|^{\frac{1}{n}}} \varphi(s) d s$; and generally $M_{\Phi}$ arises in studies of other potential operators [Ch-St-Wh].

Let $1<p, q<\infty$. We say that the inequality $P\left(M_{\Phi}, p, q, u, v\right)$ holds for a constant $C>0$ when

$$
\left\|M_{\Phi} f\right\|_{L_{u}^{q}} \leq C\|f\|_{L_{v}^{p}} \text { for all functions } f
$$

and we write $(u, v) \in A(\Phi, p, q)$ if for some constant $A>0$

$$
\Phi(Q)|Q|^{\frac{1}{Q}-\frac{1}{p}}\left(\frac{1}{|Q|} \int_{Q} u\right)^{\frac{1}{q}}\left(\frac{1}{|Q|} \int_{Q} v^{-\frac{1}{p-1}}\right)^{1-\frac{1}{p}} \leq A \text { for all cubes } Q .
$$

In this paper we always adopt the convention $0 . \infty=0 . \quad \mathrm{By}$ $P\left(M_{\Phi}, p, q, u, v\right)$ and the Lebesgue theorem, we see that if $u \neq 0$ it is necessary to suppose

$$
\lim _{|Q| \rightarrow 0}\left(\Phi(Q)|Q|^{\frac{1}{q}-\frac{1}{p}}\right)<\infty .
$$

For instance $\mathcal{H}_{3}$ is satisfied if $\frac{1}{p}-\frac{1}{q} \leq \lambda$. For $\Phi(Q)=1$, the hypothesis $\mathcal{H}_{3}$ implies $q \leq p$, and for $\Phi(Q)=|Q|^{\frac{s}{n}}$ it means $\frac{1}{p}-\frac{1}{q} \leq \frac{s}{n}$.

Let $\varrho>0$ and $w$ be a weight function. As in Section 0, we write $w d x \in B_{\varrho}$ if there is $C>0$ such as

$$
\frac{\left|Q^{\prime}\right| w}{|Q| w} \leq\left(\frac{\left|Q^{\prime}\right|}{|Q|}\right)^{\varrho} \text { for all cubes } Q, Q^{\prime} \text { with } Q^{\prime} \subset Q \text {. }
$$

Now our main result can be stated: 


\section{Theorem.}

Let $1<p, q<\infty$ and $\Phi$ be a function which satisfies $\mathcal{H}_{1}, \mathcal{H}_{2}, \mathcal{H}_{3}$.

A) If the inequality $P\left(M_{\Phi}, p, q, u, v\right)$ holds for a constant $C>0$ then $(u, v) \in A(\Phi, p, q)$ with the constant $A=C$.

B) Let $1<q<p<\infty$ and $d \sigma=v^{-\frac{1}{p-1}} d x \in A_{\infty}$. Moreover assume $u d x \in B_{\nu}, v^{-\frac{1}{p-1}} d x \in B_{\varrho}$ with $0<\nu, \varrho$ and $(1-\lambda)<\varrho\left(1-\frac{1}{p}\right)+$ $\nu\left(\frac{1}{p}\right)$. If $(u, v) \in A(\Phi, p, q)$ then the inequality $P\left(M_{\Phi}, p, q, u, v\right)$ holds for a constant $A c, c=c(\Phi, n, p, q, u, v)>0$. The part $B)$ is also valid when $d \sigma \in B_{\varrho}$ with $1-\lambda \leq \varrho$.

Actually the constant $c$ depends on the fact that $u d x \in B_{\nu}$, $v^{-\frac{1}{p-1}} d x \in B_{\varrho}$ but not directly on $u$ and $v$. The result stated in the introduction is now easily derived from the theorem by taking $\Phi(Q)=|Q|^{\frac{s}{n}}$.

Let $0<s<n$ and $I_{s}$ the fractional integral operator defined by

$$
I_{s}=\int_{\mathbb{R}^{n}}|x-y|^{s-n} f(y) d y .
$$

In the case of $1<p \leq q<\infty$ it is known $[\mathrm{Pe}]$ that the inequality $P\left(I_{s}, p, q, u, v\right)$, i.e.

$$
\left\|I_{s} f\right\|_{L_{u}^{q}} \leq C\|f\|_{L_{v}^{p}} \text { for all nonnegative functions } f
$$

holds if and only if $(u, v) \in A(s, n, p, q)$ whenever $u d x, v^{-\frac{1}{p-1}} d x \in A_{\infty}$. By the results in $[\mathbf{R a 2}]$ and $[\mathbf{R a} 3]$ this equivalence also holds if $u d x \in$ $B_{\nu} \cap D_{\infty}, v^{-\frac{1}{p-1}} d x \in B_{\varrho}$ with $1-\frac{s}{n}<\nu$ and $1-\frac{s}{n} \leq \varrho$ (see also [Pe] for such a result). The condition $w d x \in D_{\infty}$ means:

$$
|2 Q|_{w} \leq C|Q|_{w} \text { for all cubes } Q \text {. }
$$

$2 Q$ is the cube with the same center as $Q$ but the edge lenght expanded twice. As a consequence of our theorem, for $1<q<p$ we have

\section{Corollary.}

Let $1<q<p<\infty, 0<s<n$ and $u d x, v^{-\frac{1}{p-1}} d x \in A_{\infty}$. Moreover assume $u d x \in B_{\nu}, v^{-\frac{1}{p-1}} d x \in B_{\varrho}$ with $0<\nu, \varrho$ and $\left(1-\frac{s}{n}\right)<\varrho\left(1-\frac{1}{p}\right)+\nu\left(\frac{1}{p}\right)$. Then the inequality $P\left(I_{s}, n, p, q, u, v\right)$ holds if and only if $(u, v) \in A(s, n, p, q)$. This equivalence also holds when $u d x \in B_{\nu} \cap D_{\infty}, v^{-\frac{1}{p-1}} \in B_{\varrho}$ with $1-\frac{s}{n}<\nu$ and $1-\frac{s}{n} \leq \varrho$.

For seeing this, it is sufficient to remind that the MuckenhouptWheeden inequality [Mu-Wh]

$$
\left\|I_{s} f\right\|_{L_{u}^{q}} \leq C\left\|M_{s} f\right\|_{L_{u}^{q}}
$$


holds whenever $u d x \in A_{\infty}$. This is also the case when $u d x \in B_{\nu} \cap D_{\infty}$ with $1-\frac{s}{n}<\nu$ (see $[\mathbf{P e}]$ or $\left.[\mathbf{R a 2}]\right)$.

\section{On $A(\Phi, p, q)$ and $B_{\varrho}$ conditions}

Now we also assume the functions $\Phi$ defined on the set of balls by $\Phi(B)=\Phi(Q)$ whenever $Q$ is the smallest cube which contains the ball $B$. A weight function $w$ satisfies the condition $\mathcal{C}$ when there are constants $c, C>0$ so that

$$
\sup _{\frac{1}{4} R<|x| \leq 4 R} w(x) \leq \frac{C}{R^{n}} \int_{|y| \leq c R} w(y) d y .
$$

Many of usual weight functions $w$ satisfy this growth condition, since nonincreasing and nondecreasing radial functions are included. Condition $(u, v) \in A(\Phi, p, q)$ for $u$ and $v$ satisfying $\mathcal{C}$ can be easily realized, mainly for radial weights. Indeed we have

\section{Proposition 2.1.}

Let $1<p, q<\infty$ and $\frac{1}{p}-\frac{1}{q} \leq \lambda$. Assume $u, v$ satisfying the growth condition $\mathcal{C}$. Then $(u, v) \in A(\Phi, p, q)$ for a constant $A>0$ if and only if $(u, v) \in A_{0}(\Phi, p, q)$; i.e

$$
\Phi(B(0, R)) R^{n\left(\frac{1}{q}-\frac{1}{p}\right)}\left(\frac{1}{R^{n}} \int_{|y|<R} u\right)^{\frac{1}{q}}\left(\frac{1}{R^{n}} \int_{|y|<R} v^{-\frac{1}{p-1}}\right)^{1-\frac{1}{p}} \leq A_{0}
$$

for all $R>0$, where $A_{0}=A \times c(\Phi, n, p, q, u, v)$.

As an example for $0 \leq s<n, \frac{1}{p}-\frac{1}{q} \leq \frac{s}{n},-n<\alpha<n(p-1)$, $p s-n<\alpha, \beta=\frac{q}{p}(n+\alpha)-q s-n, u(x)=|x|^{\beta}, v(x)=|x|^{\alpha}$ then $(u, v) \in A(s, n, p, q)$.

Now let us discuss how we can verify in practise, for usual weights the condition $w d x \in B_{\varrho}, \varrho>0$. To do this, we first recall some known classes of weights.

\section{The Muckenhoup class $A_{p}$.}

Let us recall that $w d x \in A_{p}(1<p<\infty)$ if and only if $(w, w) \in$ $A(0, n, p, p)$. It is known [Ga-Rb] that $A_{\infty}=\cup_{r>1} A_{r}$. 
The reverse Holder class $R H_{r}$.

We write $w d x \in R H_{r}(1<r<\infty)$ if and only if

$$
\left(\frac{1}{|Q|} \int_{Q} w^{r}\right)^{\frac{1}{r}} \leq R\left(\frac{1}{|Q|} \int_{Q} w\right) \text { for all cubes } Q \quad C=C(w)>0 .
$$

The classes $R H_{r}$ and $A_{p}$ are related; for instance $w d x \in R H_{r}$ if and only if $w^{-1} d x \in A_{\frac{r}{r-1}}$. If $w d x \in A_{p}$ then it is known [Ga-Rb] that $w d x \in R H_{1+\rho}$ for some $\rho>0$; the converse is also true.

\section{The reverse doubling class $R D_{\varrho}$.}

We write $w d x \in D_{\varrho}(\varrho>0)$ if and only if

$$
C t^{n \varrho}|Q|_{w} \leq|t Q|_{w} \text { for all cubes } Q \text { and all } t \geq 1, \quad C=C(w)>0 .
$$

If $w d x \in R H_{\frac{r}{r-1}}$ then, by the Holder inequality, $w d x \in R D_{\frac{1}{r}}$. Suppose $w d x \in D_{\infty}$ with the doubling constant $D$, i.e

$$
|2 Q|_{w} \leq D|Q|_{w} \text { for all cubes } Q \quad D=D(w)>1,
$$

then [St-To] $w d x \in R D_{\varrho}$ for some $\varrho>0$. Precisely [Ra3] we can take $\varrho=\frac{1}{\ln 2^{n}} \ln \frac{D^{c}}{D^{c}-1}$ where $c=4+\frac{\ln 3}{\ln 2}$. But the reverse doubling condition $R D_{r}$ is weaker than the doubling condition $D_{\infty}$ (take for instance $w(x)=$ $\left.e^{|x|}\right)$.

Thus it is clear that $w d x \in A_{\infty}$ implies $w d x \in B_{\varrho}$ for some $\varrho$. On the otherhand we can state

\section{Proposition 2.2.}

If $w d x \in B_{\varrho}$ for some $\varrho>0$ then $w d x \in R D_{\varrho}$. Conversely if $w d x \in$ $R D_{\varrho} \cap D_{\infty}$ then $w d x \in B_{\varrho}$.

So in practice to obtain $w d x \in B_{\varrho}$ it is sufficient to get $w d x \in$ $R D_{\varrho} \cap D_{\infty}$. By the above condition $w d x \in R D_{\varrho}(0<\varrho \leq 1$, with the precise value of $\varrho$ ) can be realized from $w d x \in R H_{\frac{1}{1-\varrho}}$ or $w d x \in D_{\infty}$. Consequently, it is interesting to know when we have $w d x \in D_{\infty}$. It is well known [Ga-Rb] that $w d x \in D_{\infty}$ when $w d x \in A_{\infty}$. But we can find $w d x \in D_{\infty}$ with $w d x \notin A_{\infty}$ [Wi]. As a tool for $w d x \in D_{\infty}$ Stromberg and Wheeden [St-Wh] proved that $|x|^{\alpha} u(x),\left(\frac{|x|}{1+|x|}\right)^{\alpha} u(x) \in D_{\infty}$ when $u d x \in R D_{\varrho} \cap D_{\infty}$ and $\alpha>-n p$. By adapting an argument in [St$\mathbf{W h}$ ], this result can be extended for weights $w(x)=\theta(|x|) u(x)$ where $u d x \in R D_{\varrho} \cap D_{\infty}(\varrho>0)$, and $\theta$ essentially constant on annuli and satisfying a condition like: $\sum_{k \geq 0} 2^{-k n_{e}} \theta\left(2^{-k} L\right) \leq \theta(L)$ for all $L>0$. 


\section{Proofs of results}

Our main theorem is a direct consequence of the inequalities (3.1), (3.2), (3.3) in the following propositions.

\section{Proposition 3.1.}

Let $1<q<p<\infty$. Assuume $\Phi$ be a function which satisfies hypotheses $\mathcal{H}_{1}, \mathcal{H}_{2}, \mathcal{H}_{3}$. Let define

$$
\Theta(x)=\sup \left\{\Phi(Q)|Q|^{-1}|Q|_{\sigma}^{1-\frac{1}{p}}|Q|_{u}^{\frac{1}{p}} ; \quad Q \text { a cube with } Q \ni x\right\},
$$

$d \sigma=v^{-\frac{1}{p-1}} d x$ and $\tilde{u}(x)=\Theta^{-p}(x) u(x)$. Then $\tilde{u} \in L_{\text {loc }}^{1}\left(\mathbb{R}^{n}, d x\right)$ and

$$
\left\|M_{\Phi} f\right\|_{L_{u}^{q}} \leq\left\|M_{\Phi} f\right\|_{L_{u}^{p}}\|\Theta\|_{L_{u}^{r}} \text { for all functions } f
$$

where $r=\frac{q p}{p-q}$.

\section{Proposition 3.2.}

Let $1<p<\infty$ and $\tilde{u}$ defined as above. Assume $d \sigma \in A_{\infty}$ or $d \sigma \in B_{\varrho}$ with $1-\lambda \leq \varrho\left(\lambda\right.$ is the exponent in the hypothesis $\left.\mathcal{H}_{2}\right)$. Then there is $c=c(\Phi, n, p, q, u, v)>0$ such that

$$
\left\|M_{\Phi} f\right\|_{L_{\tilde{u}}^{p}} \leq c\|f\|_{L_{v}^{p}} \text { for all functions } f .
$$

\section{Proposition 3.3.}

Let $1<q<p<\infty$. Assume

i) $\Phi$ be a function which satisfies $\mathcal{H}_{1}, \mathcal{H}_{2}, \mathcal{H}_{3}$;

ii) $u d x \in B_{\nu}, v^{-\frac{1}{p-1}} d x \in B_{\varrho}$ with $0<\nu, \varrho$ and $(1-\lambda)<\varrho\left(1-\frac{1}{p}\right)+$ $\nu\left(\frac{1}{p}\right)$

iii) $(u, v) \in A(\Phi, p, q)$ for a constant $A>0$. Then there is $C(\Phi, n, p, q, u, v)>0$ so that

$$
\|\Theta\|_{L_{u}^{r}} \leq C A \quad r=\frac{q p}{p-q} .
$$

\section{Proof of Proposition 3.1:}

Let us first observe the locally integrability of the function $\tilde{u}$. Indeed for each cube $Q$ with $\left(\Phi(Q)|Q|^{-1}|Q|_{\sigma}^{1-\frac{1}{p}}|Q|_{u}^{\frac{1}{p}}\right)>0$ and for each $x \in Q$ :

$$
\Theta^{-1}(x) \leq\left(\Phi(Q)|Q|^{-1}|Q|_{\sigma}^{1-\frac{1}{p}}|Q|_{u}^{\frac{1}{p}}\right)^{-1}>0
$$


and so

$$
|Q|_{\tilde{u}}=\int_{Q} \Theta^{-p}(x) u d x \leq\left(\Phi(Q)|Q|^{-1}|Q|_{\sigma}^{1-\frac{1}{p}}|Q|_{u}^{\frac{1}{p}}\right)^{-p}<\infty .
$$

Note that for $|Q|_{u}=0$, by the convention $0 . \infty=0$, we immediatly have $|Q|_{\tilde{u}}=0$.

Inequality (3.1) comes from the Holder inequality, indeed for $1<q<$ $p<\infty$ and $r=\frac{q p}{p-q}$ we get

$$
\begin{aligned}
\left\|M_{\Phi} f\right\|_{L_{u}^{q}}^{q} & =\int_{\mathbb{R}^{n}}\left[\left(M_{\Phi} f\right) \tilde{u}^{\frac{1}{p}} \Theta u^{\frac{1}{q}-\frac{1}{p}}\right]^{q} d x \leq \\
& \leq\left\|\left(M_{\Phi} f\right) \tilde{u}^{\frac{1}{p}}\right\|_{L^{p}}^{q}\left\|\Theta u^{\frac{1}{q}-\frac{1}{p}}\right\|_{L^{r}}^{q}= \\
& =\left\|M_{\Phi} f\right\|_{L_{\tilde{u}}^{p}}^{q}\|\Theta\|_{L_{u}^{r}}^{q} .
\end{aligned}
$$

Proof of Proposition 3.2:

First let us note that by $(3.4),(\tilde{u}, v) \in A(\Phi, p, p)$ i.e.

$$
\left(\Phi(Q)|Q|^{-1}|Q|_{\sigma}^{1-\frac{1}{p}}|Q|_{\tilde{u}}^{\frac{1}{p}}\right)>0 \text { for all cubes } Q .
$$

For $d \sigma \in A_{\infty}$, an easy modification of the proof in $[\mathbf{P e}]$ yields to the conclusion (3.2). For $d \sigma \in B_{\varrho}$ with $1-\lambda \leq \varrho$, we get $(\tilde{u}, v) \in S(\Phi, p, p)$ [Ra3] and then by a similar argument as in [Sa2] the inequality (3.2) holds for a constant $c=c(\Phi, n, p, \tilde{u})>0$.

Proof of Proposition 3.3:

For each $R>0$, let us define $\Theta_{R}(x)=\sup \left\{\Phi(Q)|Q|^{-1}|Q|_{\sigma}^{1-\frac{1}{p}}|Q|_{u}^{\frac{1}{p}} ; Q\right.$ a cube with $\left.Q \ni x,|Q|^{\frac{1}{n}} \leq R\right\}$.

The conclusion appears once we obtain

$$
\left\|\Theta_{R}\right\|_{L_{u}^{r}} \leq c A \quad c=c(\Phi, n, p, q, u, \sigma)>0, \quad r=\frac{q p}{p-q} .
$$

Then in order to prove (3.3'), we take a cube $Q_{0}$ with $\left|Q_{0}\right|^{\frac{1}{n}}=R$. Then

$$
\|\Theta\|_{L_{u}^{r}}^{r}=\theta_{1, R}+\Theta_{2, R}
$$

where $\Theta_{1, R}=\int_{Q_{0}} \Theta_{R}^{r} u d x, \Theta_{2, R}=\int_{\mathbb{R}^{n} \backslash Q_{0}} Q_{R}^{r} u d x$. 


\section{Estimate of $\Theta_{1, R}$.}

Let $x \in Q_{0}, Q$ a cube with $Q \ni x$ and $|Q|^{\frac{1}{n}} \leq R$. Note that $Q \subset\left(3 Q_{0}\right)$. Now using i), ii), iii) we get

$$
\begin{aligned}
\Lambda(Q) & =\Phi(Q)|Q|^{-1}|Q|_{\sigma}^{1-\frac{1}{p}}|Q|_{u}^{\frac{1}{p}} \leq \\
& \leq c(\Phi, n, \sigma, u)\left(\frac{|Q|}{\left|Q_{0}\right|}\right)^{\left[\lambda-1+\varrho\left(1-\frac{1}{p}\right)+\nu \frac{1}{p}\right]} \Lambda\left(3 Q_{0}\right) \leq \\
& \leq c(\Phi, n, \sigma, u) \Lambda\left(3 Q_{0}\right) .
\end{aligned}
$$

Thus $\Theta_{R}(x) \leq c(\Phi, n, \sigma, u) \Lambda\left(3 Q_{0}\right)$, and consequently

$$
\begin{aligned}
\Theta_{1, R} & \leq c^{\prime}(\Phi, n, \sigma, u)\left(\Lambda\left(3 Q_{0}\right)\left|3 Q_{0}\right|_{u}^{\frac{1}{q}}\right)^{r}= \\
& =c^{\prime}(\Phi, n, \sigma, u)\left(\Phi\left(3 Q_{0}\right)\left|3 Q_{0}\right|^{-1}\left|3 Q_{0}\right|_{\sigma}^{1-\frac{1}{p}}\left|3 Q_{0}\right|_{u}^{\frac{1}{q}}\right)^{r} \leq \\
& \leq c^{\prime}(\Phi, n, \sigma, u) A^{r} .
\end{aligned}
$$

\section{Estimate of $\Theta_{2, R}$.}

First we can write

$$
\Theta_{2, R}=\sum_{k \geq 0} \int_{\left(2^{k+1} Q_{0}\right) \backslash\left(2^{k} Q_{0}\right)} \Theta_{R}^{r} u d x .
$$

Let $k \in \mathbb{N}, x \in\left(2^{k+1} Q_{0}\right) \backslash\left(2^{k} Q_{0}\right)$ and $Q \ni x$ with $|Q|^{\frac{1}{n}} \leq R$. Then $Q \subset\left(32^{k+1} Q_{0}\right)=\left(6 Q_{0}\right)$. As the above computation we have

$$
\Lambda(Q) \leq c^{\prime}(\Phi, n, \sigma, u) 2^{-k n\left[\lambda+\varrho\left(1-\frac{1}{p}\right)+\nu \frac{1}{p}\right]} \Lambda\left(62^{k} Q_{0}\right) .
$$

Next, since $1-\lambda<\varrho\left(1-\frac{1}{p}\right)+\nu \frac{1}{p}$, then

$$
\begin{aligned}
\Theta_{2, R} & \leq c^{\prime}(\Phi, n, \sigma, u) \sum_{k \geq 0} 2^{-k n\left[\lambda+\varrho\left(1-\frac{1}{p}\right)+\nu \frac{1}{p}\right]}\left(\Lambda\left(62^{k} Q_{0}\right)\left|\left(62^{k} Q_{0}\right)\right|_{u}^{\frac{1}{r}}\right)^{r} \leq \\
& \leq c^{\prime}(\Phi, n, \sigma, u) A^{r} \sum_{k \geq 0} 2^{-k n\left[\lambda+\varrho\left(1-\frac{1}{p}\right)+\nu \frac{1}{p}\right]} \leq \\
& \leq c^{\prime}(\Phi, n, \sigma, u) A^{r} .
\end{aligned}
$$

Inequalities (3.5) and (3.6) yield (3.3'), and consequently by a limiting argument we get (3.3). 
Proof of Proposition 2.1:

Let us assume the condition $(u, v) \in A(\Phi, p, q)$ holds for a constant $A>0$. It is also equivalent to ask

$(*)$

$$
\Phi(B)|B|^{\frac{1}{q}-\frac{1}{p}}\left(\frac{1}{|B|} \int_{B} u\right)^{\frac{1}{q}}\left(\frac{1}{|B|} \int_{B} v^{-\frac{1}{p-1}}\right)^{1-\frac{1}{p}} \leq A^{\prime} \text { for all balls } B,
$$

here $A^{\prime}=A c(\Phi, n, p, q)$.

If $\left|x_{0}\right| \leq 2 R$ then $B \subset B(0,3 R)$ and hence the first member of $(*)$ is majorized by

$$
\begin{aligned}
& c(\Phi, n, p, q) \times \\
\times & (B(0,3 R))(3 R)^{n\left(\frac{1}{q}-\frac{1}{p}\right)}\left(\frac{1}{(3 R)^{n}} \int_{|y|<3 R} u\right)^{\frac{1}{q}}\left(\frac{1}{(3 R)^{n}} \int_{|y|<3 R} v^{1-\frac{1}{p}}\right) .
\end{aligned}
$$

If $2 R<\left|x_{0}\right|$ then $2^{j} R<\left|x_{0}\right| \leq 22^{j} R$ for some $j \in N^{*}$ and hence for each $y \in B: \frac{1}{4} 2^{j} R<|x| \leq 42^{j} R$. Using the growth condition $\mathcal{C}$ for $u$ and $v^{-\frac{1}{p-1}}$ it is found $c=c(u, v)>0, C=C(u, v)>0$ such as

$$
\left(\frac{1}{|B|} \int_{B} v^{-\frac{1}{p-1}}\right) \leq C\left(\frac{1}{\left(c 2^{j} R\right)^{n}} \int_{|y|<\left(c 2^{j} R\right)} v^{-\frac{1}{p-1}}\right)
$$

and

$$
\left(\frac{1}{|B|} \int_{B} u\right) \leq C\left(\frac{1}{\left(c 2^{j} R\right)^{n}} \int_{|y|<\left(c 2^{j} R\right)} u\right) .
$$

Note that $c, C$ depend on the constants on the growth condition $\mathcal{C}$ for $u$ and $v^{-\frac{1}{p-1}}$ but not directly on these weights. Consequently in the case $2 R<\left|x_{0}\right|$, the first number of $(*)$ is now majorized by

$$
\begin{aligned}
& C(\Phi, n, p, q, c, C) 2^{-j n\left[\lambda+\frac{1}{q}-\frac{1}{p}\right]} \Phi\left(B\left(0, c 2^{j} R\right)\right) \times \\
& \times\left(c 2^{j} R\right)^{n\left(\frac{1}{q}-\frac{1}{p}\right)}\left(\frac{1}{\left(c 2^{j} R\right)^{n}} \int_{|y|<\left(c 2^{j} R\right)} u\right)^{\frac{1}{q}}\left(\frac{1}{\left(c 2^{j} R\right)^{n}} \int_{|y|<\left(c 2^{j} R\right)} v^{-\frac{1}{p-1}}\right)^{1-\frac{1}{p}}
\end{aligned}
$$

Since $\frac{1}{q}-\frac{1}{p} \leq \lambda$, we can see that inequality $(*)$ is satisfied once $(u, v) \in$ $A_{0}(\Phi, p, q)$ i.e.

$$
\Phi(B(0, R)) R^{n\left(\frac{1}{q}-\frac{1}{p}\right)}\left(\frac{1}{R^{n}} \int_{|y|<R} u\right)^{\frac{1}{q}}\left(\frac{1}{R^{n}} \int_{|y|<R} v^{-\frac{1}{p-1}}\right)^{1-\frac{1}{p}} \leq A_{0}
$$


for all $R>0$, here $A_{0}=A c^{\prime}(\Phi, n, p, q, u, v)$.

Proof of Proposition 2.2:

Let $w d x \in B_{\varrho}$ for some $\varrho>0$ i.e.

$$
\frac{\left|Q_{1}\right|_{w}}{\left|Q_{0}\right|_{w}} \leq B\left(\frac{\left|Q_{1}\right|}{\left|Q_{0}\right|}\right)^{\varrho} \text { for all cubes } Q_{0}, Q_{1} \text { with } Q_{1} \subset Q_{0} .
$$

Let $Q$ be a cube and $t \geq 1$. Taking $Q_{1}=Q$ and $Q_{0}=t Q$ we obtain

$$
t^{n \varrho}|Q|_{w} \leq R|Q|_{w}
$$

with $R=B$, hence $w d x \in R D_{\nu}$.

Conversely let $w d x \in R D_{\varrho}$ for a constant $R>0$. Also if $w d x \in D_{\infty}$ then for $Q_{1} \subset Q_{0}$ and for all cubes $Q_{2}$ having the same center as $Q_{1}$ and with $\left|Q_{2}\right|=\left|Q_{0}\right|$

$$
\begin{aligned}
\left|Q_{1}\right|_{w} & \leq R\left(\frac{\left|Q_{1}\right|}{\left|Q_{0}\right|}\right)^{\varrho}\left|Q_{2}\right|_{w} \leq \\
& \leq R\left(\frac{\left|Q_{1}\right|}{\left|Q_{0}\right|}\right)^{\varrho}\left|3 Q_{0}\right|_{w} \leq \\
& \leq R D\left|Q_{0}\right|_{w} .
\end{aligned}
$$

Here $D$ depends on the constant which is in the doubling condition for $w d x$. Consequently $w d x \in B_{\varrho}$ with the constant $B=R D$.

\section{References}

[Ch-St-Wh] S. Chanillo, J. O. Stromberg and R. L. Wheeden, Norm inequalities for potential type operators, Rev. Mat. Iberoamericana 3(4) (1987), 311-335.

[Ga-Rb] J. García-Cuerva AND J. L. Rubio de Francia, "Weighted norm inequalities and related topic," North Holland Math. Studies 116, 1985.

[Ke-Sa] R. KeRman AND E. SAWYeR, Weighted norm inequalities for potentials with applications to Scrodinger operators, Fourier transforms and Carleson measure, Ann. Inst. Fourier 36 (1986), 207-228.

[Mu] B. Muckenhoupt, Weighted norm inequalities for the Hardy maximal function, Trans. Amer. Math. Soc. 165 (1972), 207-227.

[Mu-Wh] B. Muckenhoupt And R. L. WheEden, Weighted norm inequalities for fractional integrals, Trans. Amer. Math. Soc. 191 (1974), 261-274. 
[Pe] C. PÉREz, Two weighted norm inequalities for Riesz potential and uniform $L^{p}$ weighted Sobolev inequalities, Indiana Univ. Math. J. 39(1) (1990), 31-44.

[Ra1] Y. RAKOTONDRATSIMBA, Inégalités à poids pour des opérateurs maximaux et des opérateurs de type potentiel, Thèse de Doctorat, 1991.

[Ra2] Y. Rakotondratsimba, On Muckenhoupt and Sawyer conditions for maximal operators, Publicacions Matemàtiques 37(1) (1993), 57-73.

[Sa1] E. SAWYER, Weighted norm inequalities for fractional maximal operators, Proc. CMS 1 (1981), 283-309.

[Sa2] E. SAWYER, A characterization of a two weight norm inequality for maximal operators, Studia Math. 75 (1982), 1-11.

[St-Wh] J. O. Stromberg and R. L. Wheeden, Fractional integrals on weighted $H^{p}$ and $L^{p}$ spaces, Trans. Amer. Math. Soc. 287 (1985), 293-321.

[St-To] J. O. Stromberg And A. Torchinsky, "Weighted Hardy spaces," Lecture Notes in Math. 1385, 1989.

[Ve] I. E. VERBITSKY, Weighted norm inequalities for the maximal operators and Pisier's theorem on factorization through $L^{p \infty}$, Integr. Equat. Oper. Th. 15 (1992), 124-153.

[Wi] I. WIK, On Muckenhoupt's classes of weight functions, Studia Math. 54 (1989), 245-255.

Université d'Orléans-Département de Mathématiques

U.F.R. Faculté des Sciences

B.P. 6759

45067 Orléans Cedex 2

FRANCE

Primera versió rebuda el 16 de Març de 1993, darrera versió rebuda el 3 de Febrer de 1994 